\title{
Developmental Implications of Dichotomous Ossification Sequences in the Wrist Region
}

\author{
STANLEY M. GARN, SAM T. SANDUSKY, RICHARD L. MILLER \\ AND JERROLD M. NAGY \\ Center for Human Growth and Dezelopment, The University of Michigan, \\ Ann Arbor, Michigan 48104
}

\author{
KEY WORDS Ossification order · Sex differences - Population \\ differences - Chromosomal abnormalities - Growth.
}

\begin{abstract}
As shown in radiographs of 3,764 children of European ancestry under 11 years of age, there are 55 dichotomous (present-absent/absent-present) ossification sequences for nine wrist region centers in boys and 48 such sequences for girls, with statistically significant sexual dimorphism in more than half of the "alternative" sequences. Substantial samples of Meso-American origin ("Chicanos") and largely-African origin ("Blacks") evidence additional dichotomous ossification sequences, with clear population differences, while Down's syndrome children $(47, \mathrm{G}+)$ show a major excess of the numerically rarer sequences of the wrist region.
\end{abstract}

The number of dichotomous (i.e., present-absent) ossification sequences involving 9 postnatal centers of the wrist region lies between the theoretical minimum of 36 and the numerical maximum of 72 . The minimum estimate is tenable only if there is no departure from the median ossification sequence, such that center A always precedes center $B$, etc., while the maximum number is attainable only if all possible dichotomous ossification sequences exist, B-A, C-A, D-A, etc. Between the theoretical minimum of $\frac{\mathrm{N}(\mathrm{N}-1)}{2}$ sequences and the theoretical maximum, calculated as $\mathrm{N}(\mathrm{N}-1)$, lies the real world of ossification variability and its implications. Contained between the numerical limits given above are such problems as the quantification of sexual dimorphism in ossification order, the magnitude of population differences, possible effects of nutritional extremes, and finally, the diagnostic implications of ossification order.

As a first step in delineating the number of dichotomous ossification sequences for the wrist-area centers, we have turned to recently-compiled ossification data on 3,764 subjects of European derivation, comprising 1989 boys and 1775 girls. The study was restricted to those subjects exhibiting at least one wrist-area center in standardized postero-anterior radiographs, but not more than eight of the nine wristarea centers. Ossification data were initially recorded on optical scanning cards, then transferred to 80 column punchcards, and finally to magnetic tape. After verification, a special computer program was used to prepare $9 \times 9$ matrices showing the number and percent of cases evidencing each of two possible sequences for any given pair of centers. The readout and the data analysis were strictly dichotomous, i.e., present/absent, and no size interpolations were allowed.

As shown in the tabular matrix reproduced in table 1, containing the frequency of each possible dichotomous sequence for all nine wrist-area centers, the actual number of dichotomous sequences is more than the theoretical minimum of 36 and less than the numerical maximum of 72 . To be specific, there were 55 different sequences for boys - the minimal 36 shown on the right-hand side of the diagonal, and 19 additional alternative sequences (on the left). For girls, a total of 48 different dichotomous sequences was observed, the minimal 36 on the righthand side of the diagonal, and 12 additional "alternative" dichotomous sequences as shown in the left-hand side of the diagonal. 

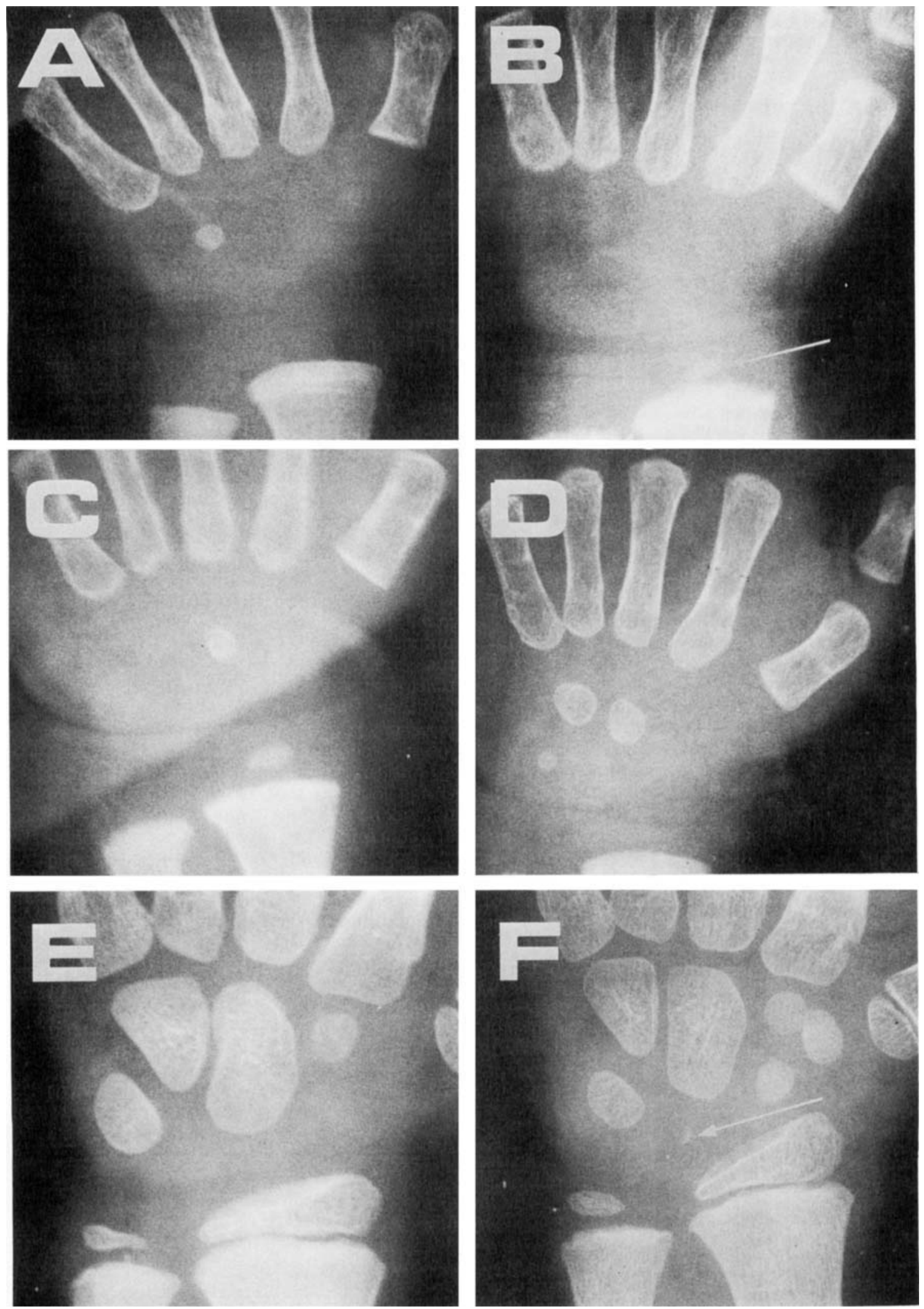

Figure 1 
While some dichotomous ossification sequences were in fact invariable, such as capitate-distal ulna, it is impressive how many different sequences were found, often well out of the expected order. Among these may be included the lunatedistal radius sequence, the scaphoid-triquetral sequence, the trapezium-triquetral sequence - all in boys, and the distal ulna-lunate sequence in both sexes. As shown in the table and as pictured in figure 1 , no single center is completely invariable in ossification sequence, some centers participate in a wide variety of dichotomous (present-absent) ossification sequences, and there are situations where the distal radius actually precedes the capitate and hamate. Clearly there is wide variety in the programming of developmental events, halfway to the theoretical maximum.

While the sex difference in the number of dichotomous ossification sequences is not viewed as necessarily significant, in view of the differential numbers of subjects considered in the sampling, many of the sexual dimorphisms in the frequencies of different orders are significantly different from zero, by both Npq and chi-squared tests. For example the trapezium-scaphoid order appears in 54\% of girls but only $39 \%$ of boys. The trapezoid precedes the trapezium in $58 \%$ of boys but only in $45 \%$ of girls. The distal ulna is earlier than the trapezium in $15 \%$ of boys and $6 \%$ of girls. Out of 19 "alternative" sequences enumerated on the left of the diagonal in table 1 , eight (denoted by asterisks) were significantly different in frequency between boys and girls. Clearly the sexes differ in the relative order of ossification events, to a far larger

Fig. I Rare or uncommon dichotomous ossification sequences of the wrist area and their frequencies $(f)$ in six children of European ancestry. (A) female showing the less-common hamatecapitate ossification order $(f=0.05)$. (B) male, showing the distal radius (arrow) earlier in appearance than either the capitate or the hamate $(f=0.02)$. (C) the capitate and distal radius both ossified prior to the hamate ( $f=0.05$ in males) (D) the capitate, hamate and triquetral ahead of the distal radius (male, $f=0.02$ ). (E) distal ulna ahead of the lunate, scaphoid and trapezium. The rare distal ulna-lunate sequence is found in only $1 \%$ of males or females $(f=0.01)$, (F) illus trating the lunate (designated by arrow) later in appearance than the distal ulna, again a rare sequence $(f=0.01)$. degree than would be expected by chance alone.

Besides these sex differences in the frequency of different dichotomous sequences for the basic series of 3,764 boys and girls of European derivation, there are also population differences that merit mention. Among 299 girls of Meso-American ancestry ("Chicanos"), similarly studied, there were numerous differences in the order of wrist-area centers, including a notably large proportion $(69 \%)$ with the trapezoid-scaphoid order as compared with $49 \%$ in the girls of European derivation. Out of 1156 Black or American Negro girls and 1084 Black or American Negro boys, there were many divergences from table 1, including the distal radius-hamate order, the lunate-distal radius order, and the lunate-triquetral order in up to $10 \%$ of Black girls. A pilot series of 75 Iranian boys (from near Shiraz) evidenced the trapezium-lunate, trapezoid-lunate, and distal ulna-lunate orders - as part of a syndrome of lunate lateness. Dichotomous ossification sequences evidently differ from population to population as well as between the sexes when the additional 2614 children are taken into account.

Besides these differences in dichotomous ossification sequences that relate to sex or geographical ancestry, a preliminary review of 56 Down's syndrome boys and girls with the 47,G + karyotype showed an excess of the rarer sequences to the left of the diagonal - such as triquetraldistal radius, scaphoid-lunate, and trapezium-lunate, for some orders remarkably so. The present method of comparison also documents the ossification irregularity in homocystinuria, previously discussed by us (Poznanski et al.,'71).

Now the data in table 1 present for the first time an estimate of the sex-specific frequencies of different dichotomous ossification orders in an extended population sample. They confirm and extend knowledge of ossification sequence variability previously reported by us from more limited longitudinal data (Garn and Rohmann, '60; Garn, Rohmann and Blumenthal, '66; Garn, Rohmann and Silverman, '67). They amplify the analysis of triquetral variability reported by Johnston, Whitehouse and Hertzog, in 1968. They suggest that when 28-29 hand-wrist centers are similarly 


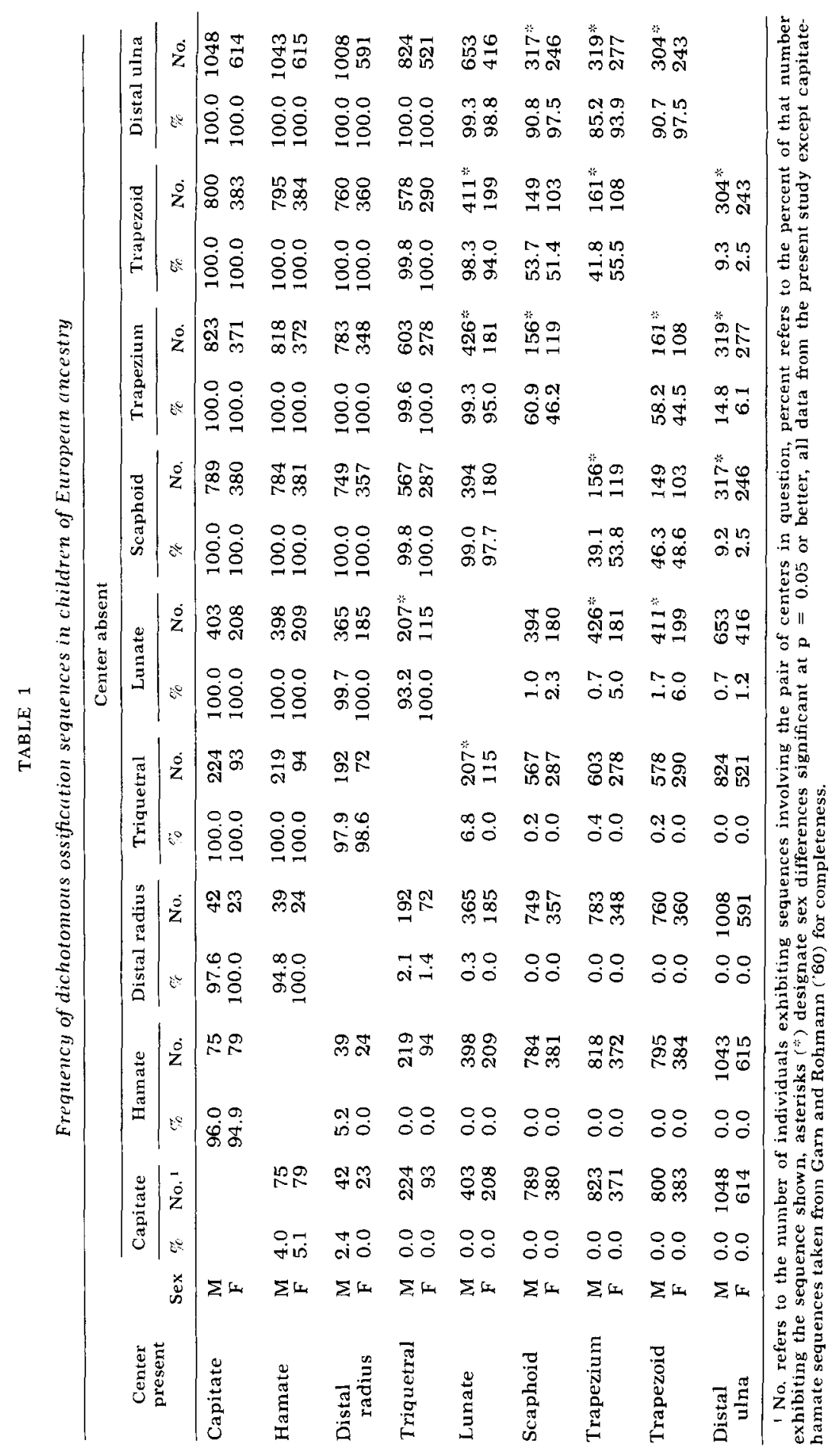


analyzed in complete matrix form that there will be some 675 different dichotomous ossification orders, further attesting to the variability of human development, even in a single population sample representing less than half of the socio-economic range.

These findings show the extent of developmental differentiation between the sexes, such that the order of developmental events differs markedly between boys and girls. They show that an extra Ggroup chromosome affects developmental order as well as developmental timing. With reasonable population estimates as to the frequency of different dichotomous ossification sequences and a useful paradigm, it is now possible to ascertain whether malnutrition and overnutrition affect timing alone or both timing and the order of developmental events.

\section{ACKNOWLEDGMENTS}

The present data include 2,240 children of largely-African origin and 506 boys and girls of Meso-American origin collected during the 10-State Nutrition Sur- vey of 1968-1970 and analyzed under Contract No. HSM 21-72-522. We appreciate the assistance of Marcia L. Lux and Nancy $M$. Rosen in phases of the data analysis, permission of Dr. James A. Halsted to cite findings on Iranian children and the efforts of Shirley M. Garrett in the manuscript preparation.

\section{LITERATURE CITED}

Garn, S. M., and C. G. Rohmann 1960 Vari ability in the order of ossification of the bony centers of the hand and wrist. Am. J. Phys. Anthrop., 18: 219-230.

Garn, S. M., C. G. Rohmann and T. Blumenthal 1966 Ossification sequence polymorphism and sexual dimorphism in skeletal development. Am. J. Phys. Anthrop., 24: 101-115.

Garn, S. M., C. G. Rohmann and F. N. Silverman 1967 Radiographic standards for postnatal ossification and tooth calcification. Med. Radiog. Photog., 43: 45-66.

Johnston, F, E., R. H. Whitehouse and K. P. Hertzog 1968 Normal variability in the age and first onset of ossification of the triquetral. Am. J. Phys. Anthrop., 28: 97-99.

Poznanski, A. K., S. M. Garn, L. R. Kuhns and S. T. Sandusky 1971 Dysharmonic maturation of the hand in the congenital malformation syndromes. Am. J. Phys. Anthrop., 35: 417-432. 